Document downloaded from:

http://hdl.handle.net/10251/60868

This paper must be cited as:

Areias, A.; Ribeiro, C.; Sencadas, V.; Garcia Giralt, N.; Diez Perez, A.; Gómez Ribelles, JL.; Lanceros Mendez, S. (2012). Influence of crystallinity and fiber orientation on hydrophobicity and biological response of poly(L-lactide) electrospun mats. Soft Matter. 8(21):5818-5825. doi:10.1039/c2sm25557j.

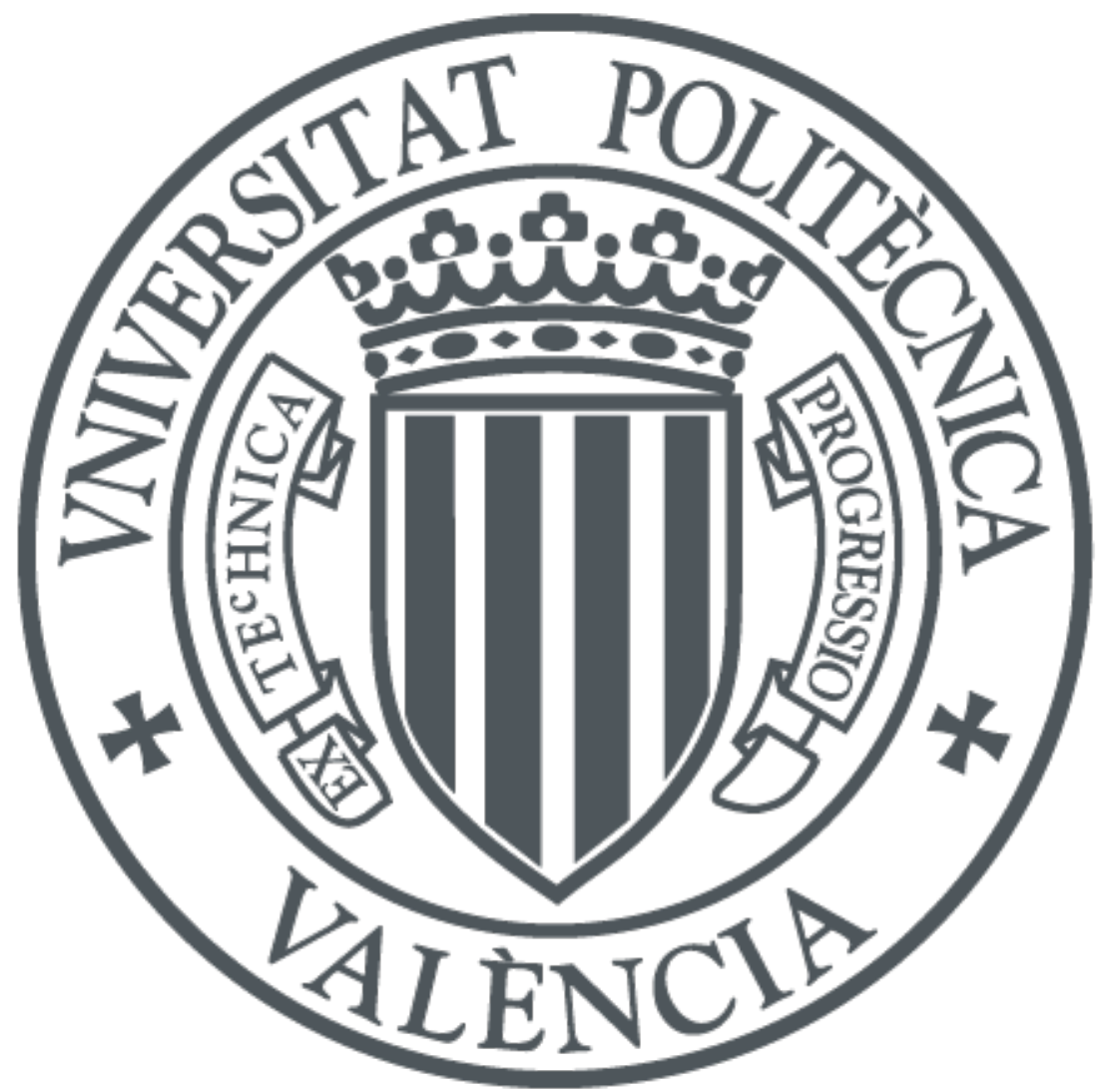

The final publication is available at

http://dx.doi.org/10.1039/C2SM25557J

Copyright Royal Society of Chemistry

Additional Information 


\title{
Influence of crystallinity on hydrophobicity and biological response of poly(L-lactide) electrospun mats.
}

A.C. Areias ${ }^{1}$, C. Ribeiro ${ }^{1}$, V. Sencadas, ${ }^{1,2}$, N. Garcia-Giralt ${ }^{5}$, A. Diez-Perez ${ }^{5}$, J.L. Gómez Ribelles ${ }^{3,4,}$ and $S$. Lanceros-Méndez ${ }^{1, *}$

${ }^{1}$ Centro/Departamento de Física da Universidade do Minho,Campus de Gualtar, 4710057 Braga, Portugal

${ }^{2}$ Instituto Politécnico do Cávado e do Ave, Campus do IPCA, 4750-810, Barcelos, Portugal.

${ }^{3}$ Center for Biomaterials and Tissue Engineering, Universitat Politècnica de València, Camino de Vera s/n, 46022 Valencia, Spain

${ }^{4}$ Networking Research Center on Bioengineering, Biomaterials and Nanomedicine (CIBER-BBN), Valencia, Spain

${ }^{5}$ URFOA, IMIM, Parc de Salut Mar, RETICEF, Barcelona, Spain

[*] Corresponding author; lanceros@fisica.uminho.pt

Tel: 3512536040 73; Fax: 351253604061

\begin{abstract}
Poly(L-lactide) electrospun mates have been produced with random and aligned fiber orientation and degrees of crystallinity from 0 up to nearly $50 \%$. These two factors, fiber alignment and degree of crystallinity strongly affect the hydrophobicity of the samples, being this larger for the aligned fiber mats and for the fibers with higher degree of crystallinity. Whereas the first effect can be associated to a decrease in the degree of porosity the second should be related to variations in the fiber roughness at nanometric scale and an increase in fiber stiffness. Proliferation of human chondrocytes cultured in monolayer on these substrates is similar in both aligned and non-aligned amorphous mats. Crystallization of the aligned mats, on the other hand, nearly suppresses proliferation and the cells produce higher amounts of aggrecan, characteristic of the extracellular matrix of hyaline cartilage.
\end{abstract}

Keywords: Nanofibers, electrospinning, PLLA, Chondrocytes, Tissue engineering 


\section{Introduction}

The use of tissue engineering for restoring, maintaining or enhancing tissue and organ function is a rapid growing field of investigation [1] with important achievements both at "in vitro" and "in vivo" experimental levels. An important goal in this field is to improve the success rate of tissue engineering strategies by creating more sophisticated materials which can mimic the extra-cellular matrix (ECM), so that the body regenerates "neo-native" functional tissues [2]. One of the major challenges in tissue engineering is articular cartilage regeneration as current regeneration therapies fail producing functional hyaline cartilage and the regenerated tissue has the characteristics of fibrocartilage [3].

Biomaterials which mimic the ECM such as nanofibers have been pointed out as good scaffolds for cartilage regeneration [4]. ECM typically consists on a viscoelastic network with nanofibrous proteins that provide biological and chemical moieties as well as physical framework supporting cell attachment and growth [5]. Chondrocytes seeded on a scaffold should produce their own highly hydrated ECM which consists mainly in collagen fibrils, predominantly collagen type II, and proteoglycans [6]. These products will maintain the structural and functional integrity of chondrocytes such as shape, polarity, migration, differentiation, apoptosis and gene expression. Cartilaginous matrix is biosynthesized during the chondrogenic differentiation and can thus be used as a marker of their differentiation [7-8].

Poly(L-lactide) (PLLA) is a biodegradable and biocompatible material approved by U.S. Food and Drug Administration (FDA) for human clinical applications [9]. This polymer shows a wide range of applications and can be processed in a variety of ways and in different forms. PLLA nanofibers produced by electrospinning, the most common and versatile method used to produce nanofibers, have a large interest due to their structural similarity to the extracellular matrix of biological systems such as collagen fibers [10]. High surface area to volume ratio and the characteristics described above indicates that it may serve as effective tissue engineering scaffolds [11].

Electrospinning originates nanofibers with different morphology and hydrophobicity. Recent findings showed that mammalian cells do respond to nanosized features influencing the cellular behavior, as for example, cell adhesion, proliferation, matrix production, cell morphology and orientation [9, 12-15]. Increasing evidences show that surface patterning or alignment of nanofibers influences the guide cell growth direction 
and morphology [5, 16-18]. Zhang et al. [18] show the contact guide of OP9 stroma cells on grooves patterned in PDMS surface. The cell movement velocity and cell number inside the microchannel, consisting in aligned nanofibers, influenced the cells migratory ability. Further, the surface topography can induce the cells to change their genetic code leaving them on tumor cell metastic form.

Several articles reported contradictory results about the effects of material topography, hydrophobicity and wettability on cell behaviour [19]. Some correlations between the wettability and cell behavior have been reported showing that cells prefer to attach on hydrophilic surfaces. Further studies found that cells adhere, spread and grow more easily on substrates with moderate hydrophilicity [20]. To improve biomaterial surfaces to make them more suitable to cell attachment and spreading, methods such as enriching surfaces with ECM components or incorporation of natural polymers with synthetic polymers have been reported. Cui et al [21] studied the effects of different biomimetic surfaces of poly (L-lactic acid) on articular chondrocytes in vitro in order to improve cytocompatibility. Gelatin was used to modify PLLA film surfaces by two different processes: physical entrapment and chemical coupling. The obtained surfaces showed different hydrophobicity and cell adhesion. Proliferation and differentiation were more efficient on the more hydrophilic surfaces which correspond to the PLLA films treated by chemical coupling. Similar works with electrospun PLLA nanofibers modified with cationized gelatin (CG) show the affinity of chondrocyte for the modified form instead of the virgin form. The chondrocytes seeded in nanofiber PLLA membranes displayed a dedifferentiated, fibroblast - like morphology, whereas chondrocytes seeded in CG PLLA NFM maintained their chondrocytic phenotype.

The goal of this work is evaluate the human articular chondrocyte behavior seeding on PLLA nanofiber scaffolds with different degree of crystallinity in order to assess the optimal environmental conditions for regenerate hyaline cartilage. Cell adhesion, proliferation and differentiation of chondrocytes, as well as their morphology and ECM production detection were studied in an in vitro environment.

\section{Materials and Methods}




\subsection{Preparation of PLLA electrospun membranes}

Biodegradable poly(acid L-lactide) electrospun membranes were prepared by electrospinning under the conditions indicated in [22]. Alignment of the fibril mats was obtained using a rotating collector while randomly oriented fiber mats were obtained with a flat collector. The mean diameter of the fibrils was $700 \mathrm{~nm}$ (standard deviation $\pm 235 \mathrm{~nm})$. The polymer fibers obtained after electrospinning were nearly amorphous, as determined by DSC thermograms. Further isothermal annealing at temperatures in the range between 70 and $140{ }^{\circ} \mathrm{C}$ allows controlled crystallization of the polymer without disturbing fibrils morphology [22]. In this way, oriented and non-oriented mats with approximately $0 \%, 8 \%, 27 \%$ and $50 \%$ of crystallinity were produced. The different PLLA samples used in present work are designed as listed in Table 1.

For cell culturing, circular samples with 6-7 $\mathrm{mm}$ of diameter of oriented and nonoriented membranes with different degrees of crystallinity were cut and sterilized with ethylene oxide. PLLA samples were hydrated by Hanks' balanced salt solution (Sigma Aldrich) overnight before cell culture and after that the samples were washed 1 time for 5 minutes in Dulbecoo's Modified Eagle's Medium (DMEM, Gilco, Invitrogen, Paisley, Scotland, UK).

Table 1 - Specification of the PLLA samples.

\begin{tabular}{|c|c|c|}
\hline Morphology of PLLA fibers & Crystallinity & Symbol \\
\hline \multirow{3}{*}{ Non-oriented } & Amorphous & NO-PLLA-0 \\
\cline { 2 - 3 } & $8 \%$ & NO-PLLA-8 \\
\cline { 2 - 3 } & $27 \%$ & NO-PLLA-27 \\
\cline { 2 - 3 } & $50 \%$ & NO-PLLA-50 \\
\hline \multirow{3}{*}{ Oriented } & Amorphous & O-PLLA-0 \\
\cline { 2 - 3 } & $8 \%$ & O-PLLA-8 \\
\cline { 2 - 3 } & $27 \%$ & O-PLLA-27 \\
\cline { 2 - 3 } & $50 \%$ & O-PLLA-50 \\
\hline
\end{tabular}

\subsection{Contact angle measurements}

Contact angle measurements (sessile drop in dynamic mode) were performed at room temperature in a Data Physics OCA20 device using ultrapure water as test liquid. The contact angles were measured by depositing water drops $(3 \mu \mathrm{L})$ on the sample surfaces using the software SCA20. At least 6 measurements in each PLLA electrospun 
membranes were performed in different sample locations and the average contact angle was taken for each sample.

\subsection{Cell culture}

Human primary chondrocytes were obtained from cartilage specimens extracted from knee samples otherwise discarded at the time of arthroplasty surgery in postmenopausal woman, as described previously $[8,23]$. Briefly, the articular cartilage was separated from bone, cut into small fragments and treated with hyaluronidase at $0.5 \mathrm{mg} / \mathrm{ml}$, pronase at $1 \mathrm{mg} / \mathrm{ml}$ and collagenase at $0.5 \mathrm{mg} / \mathrm{ml}$. The digested cartilage was filtered with $70 \mu \mathrm{m}$ of pore size, centrifuged and finally placed on a tissue culture flask with Dulbecco's Modified Eagle medium (DMEM) supplemented with 10\% fetal bovine serum (FBS) and $50 \mu \mathrm{g} / \mathrm{ml}$ ascorbic acid. At confluence, the cells were subcultured maximum twice to obtain the desired cell number. The cells that were not used immediately in experiments were frozen in liquid nitrogen with $10 \%$ dimethyl sulfoxide (DMSO) until use.

Circular membranes of PLLA microfibers were placed in a 96-multi well plate and cultured with 140000 cell/well in DMEM medium supplemented with 10\% FBS and 50 $\mu \mathrm{g} / \mathrm{ml}$ ascorbic acid at $37{ }^{\circ} \mathrm{C}$ and $5 \% \mathrm{CO}_{2}$. After two days of culture, the samples were changed to new wells and cultured with DMEM supplemented with 1\% of ITS (Insulin, Transferin and Selenium; BD Biosciences), $50 \mu \mathrm{g} / \mathrm{ml}$ ascorbic acid and 10ng/ml of transforming growth factor beta 1 (TGF $\beta 1$ ), corresponding this step to the zero time. The cell culture medium was replaced every 3 days during 28 days. The negative control was the biomaterial without cells and cells cultured on tissue culture polystyrene (TCPS) was used as a positive control.

\subsection{Cell morphology}

At 21 days of culture, chondrocytes were washed in Dulbecco's Phosphate Buffered saline (DPBS, Invitrogen) and fixed in $4 \%$ formaldehyde solution (Sigma) in PBS during 10 minutes at room temperature. The samples were then rinsed with DPBS and permeabilized with $0.1 \%$ Triton $\mathrm{X}-100$ in PBS during 3 to 5 minutes at room temperature. Then, the samples were incubated with Alexa Fluor ${ }^{\circledR} 488$ phalloidin (Invitrogen) at $6.6 \mu \mathrm{M}$ during 20 minutes. Finally, samples were washed with PBS before being mounted in a coverslip with aqueous mounting medium containing DAPI 
(Invitrogen). Overall cell morphology was studied using a confocal laser scanning microscope (Leica TCS SP5 CFS) with an excitation at $495 \mathrm{~nm}$ and emission at $518 \mathrm{~nm}$. After cell proliferation for the desired period of culture, all samples were washed with PBS solution to remove the non-adherent cells and fixed with $0.25 \%$ of glutaraldehyde for $1 \mathrm{~h}$ at room temperature. Following, the samples were dehydrated through a series of graded alcohol series. The dried scaffolds were observed in SEM equipment (Leica Cambridge S360) at an accelerating voltage of $15 \mathrm{kV}$.

\subsection{Cell viability and proliferation}

Cell viability assay and proliferation analysis was performed at $0,7,14$ and 28 days of culture on the material by MTT Kit (Invitrogen ${ }^{\mathrm{TM}}$ ) and Live/Dead Viability/Cytotoxicity Kit (Molecular Probes ${ }^{\mathrm{TM}}$ ).

MTT is an assay based on the cleavage of the tetrazolium salt in the presence of an electron coupling reagent intracellular in viable cells. The amount of formazan dye formed is directly correlated to the number of metabolically active cells in the culture and was measured at $550 \mathrm{~nm}$ using a plate reader (Biochrom Anthos 2010 Microplate Reader).

The Live/Dead Viability/Cytotoxicity Kit, which provides two color fluorescence cell staining, is based on the simultaneous determination of live and dead cells measuring recognized parameters of cell viability; intracellular esterase activity and plasma membrane integrity. The assay was evaluated by Confocal Fluorescent Microscope (Leica TCS SP5 CFS).

\subsection{Cell differentiation}

The chondrocyte differentiation was determined using enzyme-linked immunosorbent assay (ELISA) and immunohistochemical methods.

\subsubsection{Human aggrecan}

The quantitative measurement of human aggrecan (AGG) was evaluated at 7, 14 and 28 days by means of ELISA (KAP1461, DIAsource ImmunoAssays S.A., Belgium) in cell culture supernatants from experimental wells according to the manufacture protocol. The absorbance was measured at $450 \mathrm{~nm}$ by using a plate reader (Biochrom Anthos 2010 Microplate Reader). 


\subsubsection{Collagen type II}

Specimens were fixed with 50/50 of ethanol/acetone during 10 minutes at 7 and 21 days of culture. The samples were rehydrated through washing with PBS during three times and then incubated with blocking solution (10\% FBS, 0.1\% Triton x-100 in PBS) at room temperature. Then, the samples were incubated with a mouse antibody anticollagen type II (Ab-1), $1 \mu \mathrm{g} / \mathrm{ml}$ (Calbiochem $\left.{ }^{\circledR}\right)$ during 1 hour. The samples were washed with PBS and incubated with a secondary antibody Alexa Fluor ${ }^{\circledR} 488$ antimouse (Invitrogen) (1:200). . Finally, samples were washed 2 times for removing the excess of antibody before being mounted in a coverslip with mounting medium containing DAPI staining (Invitrogen). The assay was performed at Confocal Fluorescent Microscope (Leica TCS SP5 CFS) (ex/em 495 nm/ 515 nm for green fluorescence and ex/em $\sim 358 \mathrm{~nm} / \sim 461 \mathrm{~nm}$ for DAPI fluorescence).

\subsection{Statistical Analysis}

All quantitative results were obtained from triplicate and were expressed as mean \pm standard deviation. Statistical differences were determined by ANOVA using F-test for the evaluation of different groups. $\mathrm{P}$ values $<0.05$ were considered to be statically significant.

\section{3 .Results}

\subsection{Contact angle measurements}

The wettability of different PLLA membranes was determined and is shown in figure 1. The amorphous non-oriented PLLA membrane shows to be the most hydrophilic sample, with a water contact angle (WCA) of $65,9^{\circ}$. The annealing treatment of nonoriented PLLA membranes produces a decrease of wettability in the surface of these samples, at the same time than crystallinity increases. In this way, a WCA of $118,1^{\circ}$ was obtained for the NO-PLLA-50. A significant difference between oriented and nonoriented PLLA membranes for the same degree of crystallinity was observed. 


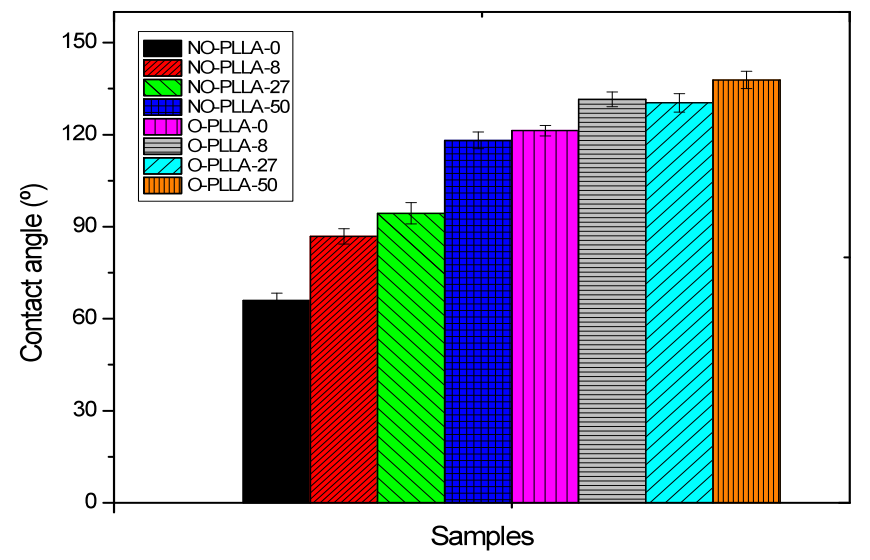

Figure 1 - Evaluation of water contact angle of PLLA membranes. Values are mean \pm SD. No statistically significant difference in contact angle was found between nonoriented PLLA membrane with $50 \%$ of crystallinity and oriented PLLA membrane amorphous and also between oriented PLLA membrane with $8 \%$ and $27 \%$ of crystallinity.

Annealing oriented PLLA membranes also affects the wettability of the samples, but in a less effective way than in the non-oriented PLLA membranes is smaller. In this way, the WCA of O-PLLA- 0 is $121,3^{\circ}$ and for O-PLLA-50 is $137,8^{\circ}$.

It is important to notice in this sense that the annealing treatment does not change the morphology of the fiber mats [22], being the variations of the wettability to be fully ascribed to variations in the nanotopography of the fiber and in the stiffness due to the increase of the degree of crystallinity.
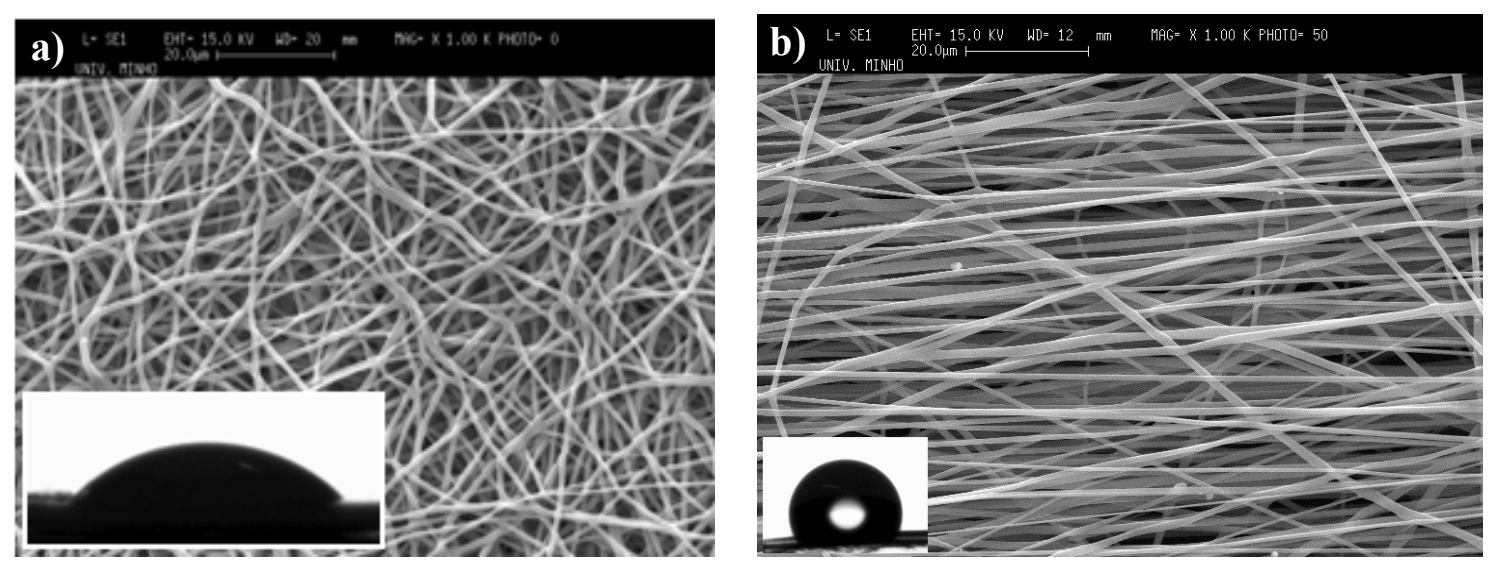

Figure 2 - a) Micrograph of static contact angle of non-oriented PLLA amorphous and

b) Micrograph of static contact angle of oriented PLLA amorphous. 


\subsection{Cell attachment and cell morphology}

The overall morphology of chondrocytes seeded in PLLA membranes after 7, 14 and 28 days was visualized by SEM (figure 3) and after 21 days in confocal fluorescence microscopy after actin staining (figure 4). According to the evaluation form results similar to the ones presented in figure 3, a higher density of cells was observed on scaffolds at 14 days of cell culture independently of the degree of crystallinity of the membranes.
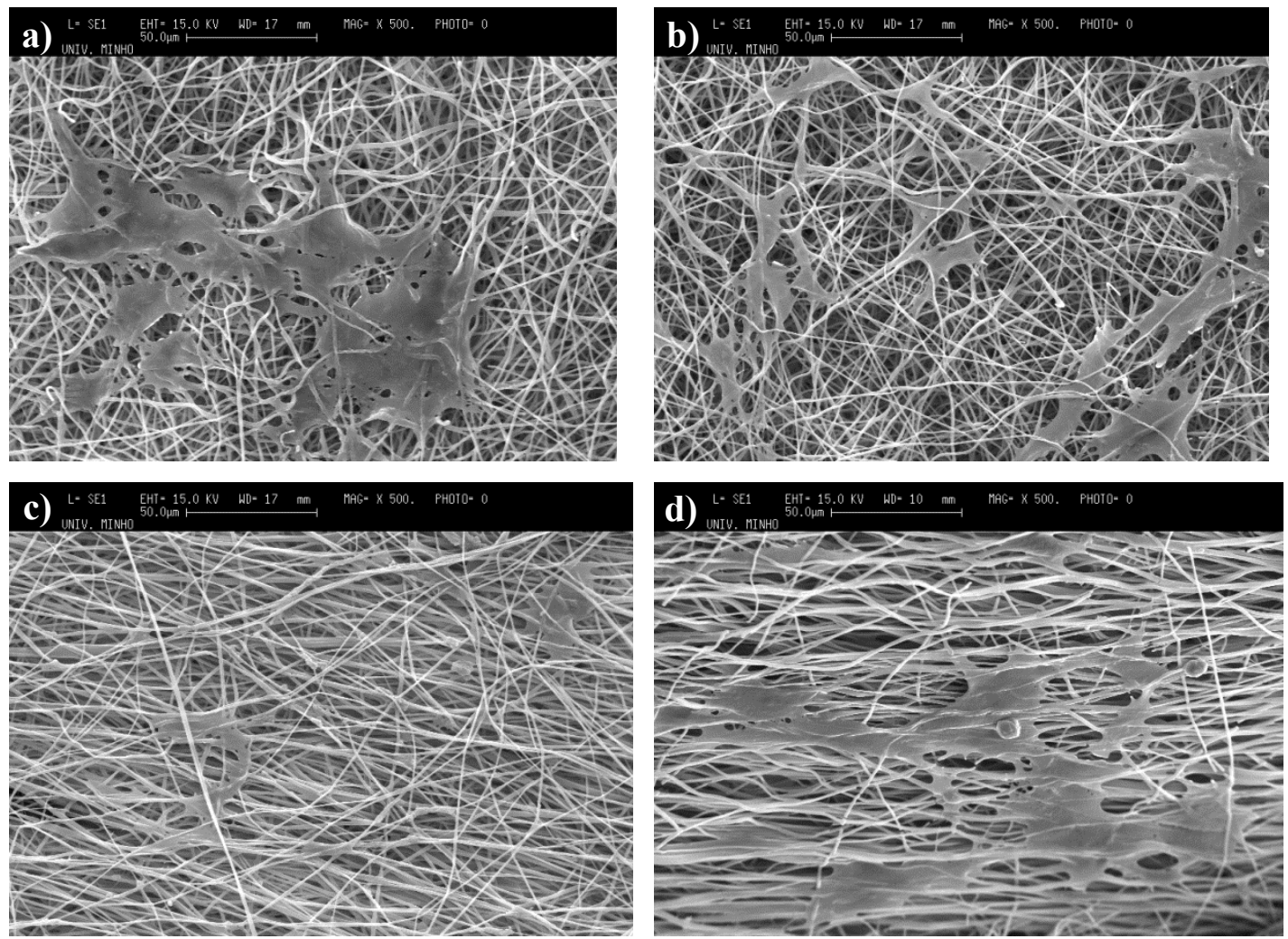

Figure 3 - Overall cell morphology of chondrocytes on PLLA membranes analyzed by SEM. a) amorphous non-oriented PLLA membrane for 14 days; b) non-oriented PLLA membrane with $27 \%$ of crystallinity for 7 days; c) oriented PLLA membrane with $27 \%$ of crystallinity for 7 days; d) oriented PLLA membrane with $27 \%$ of crystallinity for 14 days. The scale bar $(50 \mu \mathrm{m})$ is valid for all the images.

With respect to the comparison of cell morphology between oriented PLLA membranes and non-oriented PLLA membranes, it was observed that cells exhibited a more elongated morphology in oriented PLLA scaffolds (Figure 4). However, no significant 
differences were observed among cell cultures for a given fiber orientation of PLLA membranes with different degrees of crystallinity (data not shown).
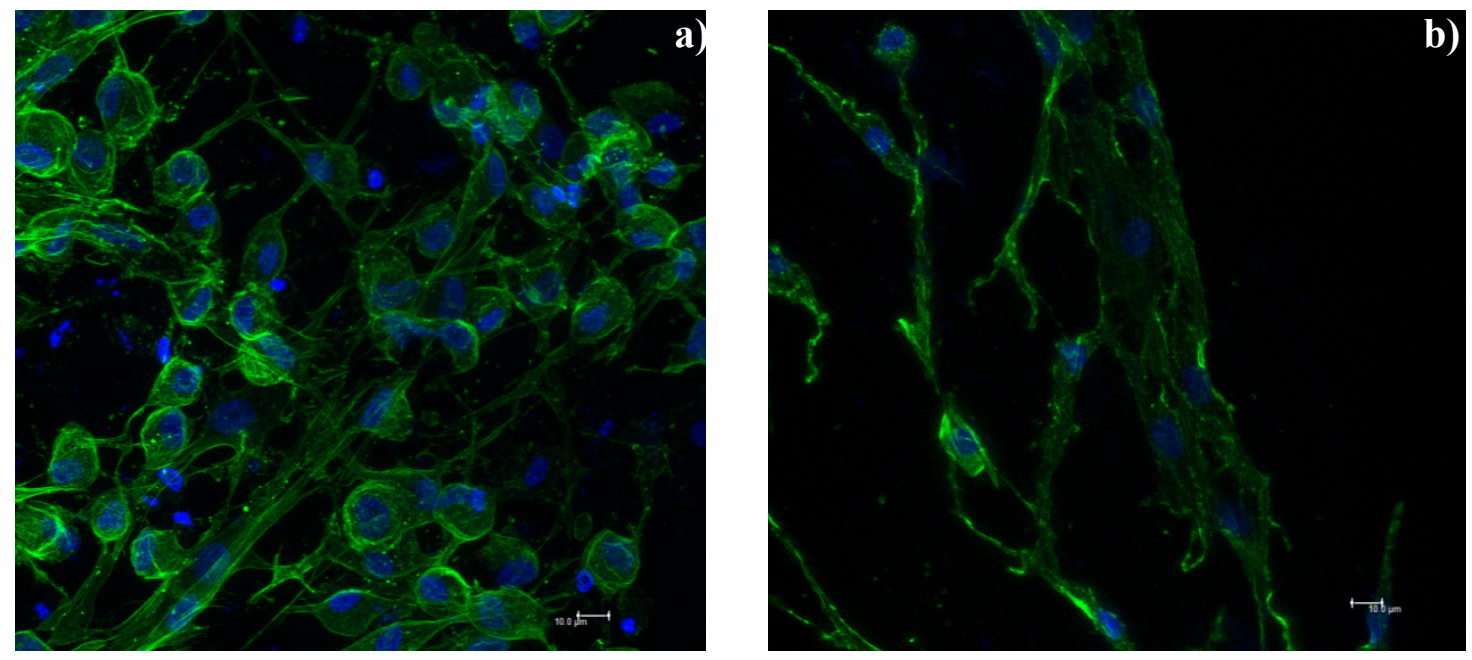

Figure 4 - Confocal fluorescence microscopy images of chondrocyte cells after 21 days of cell culture in PLLA membranes. a) non-oriented PLLA membrane amorphous; b) oriented PLLA membrane amorphous. The scale bar $(10 \mu \mathrm{m})$ is valid for all the images (green: cytoskeleton; blue: nucleus).

\subsection{Cell viability and proliferation}

The proliferation and viability of the attached cells in the eight types of PLLA membranes after 7, 14 and 28 days in culture is shown in figure 5. For all the membranes, the viable cell number increased to 14 days of cell culture and decreased for longer cell culture time.

After 7 days of cell culture, cell viability and proliferation are similar for all the evaluated samples with a slight increase compared to time 0 . At 14 days of cell culture, the amorphous PLLA membranes promote higher proliferation compared to crystalline PLLA membranes. Among crystalline PLLA membranes, cell viability is significantly lower in the case of the oriented PLLA membranes. Interestingly, the viability of the attached cells of the non-oriented PLLA membranes was higher in PLLA membranes with $27 \%$ of crystallinity than PLLA membranes with $8 \%$ and $50 \%$ of crystallinity. On the oriented PLLA membranes no significant differences are observed with the increase of crystallinity. 


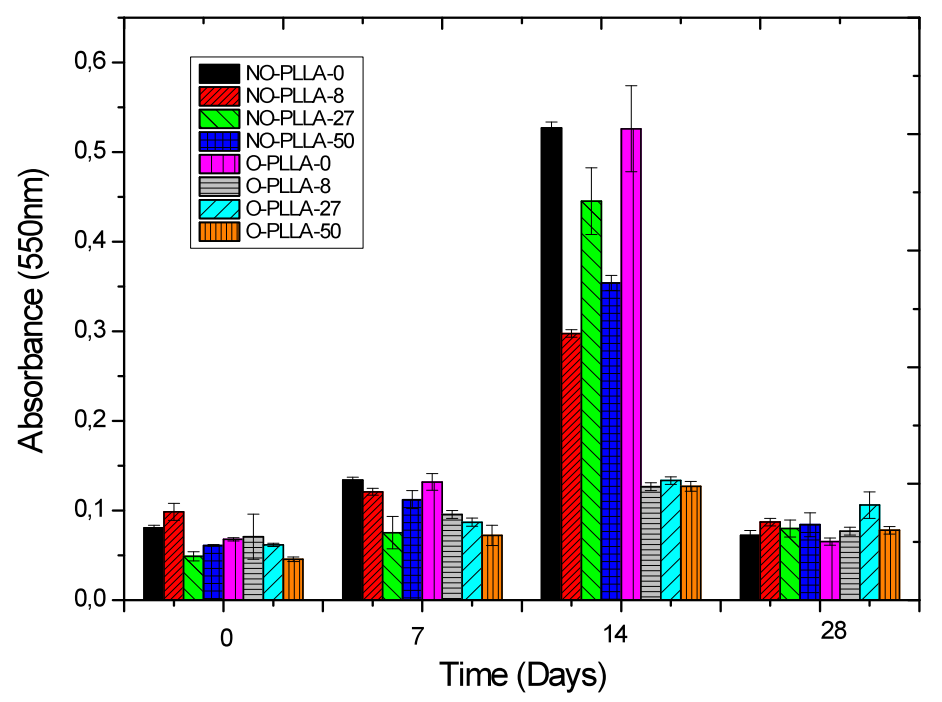

Figure 5 - MTT absorbance results after cells seeded for 0, 7, 14 and 28 days on different PLLA membranes. Values are mean \pm SD. *Significantly different $(p<0.05)$ PLLA samples.

To verify the viability of the chondrocytes on the material, a live/dead assay was also performed after 21 days of cell culture, confirming that most of adherent cells were viable (figure 6).
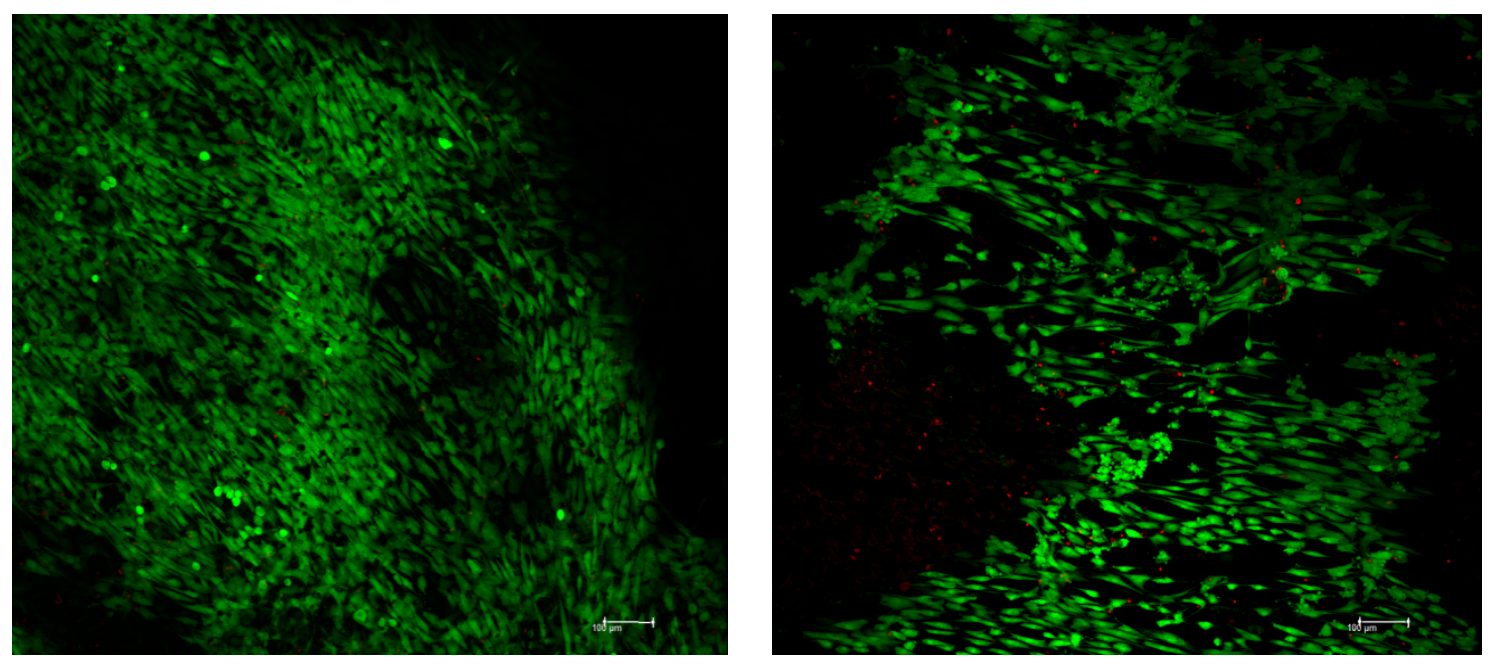

Figure 6 - Fluorescence micrographs of live/dead of cell culture with chondrocyte cells during 21 days in PLLA membranes. a) Non-oriented amorphous PLLA membrane; b) oriented amorphous PLLA membrane. The scale bar $(100 \mu \mathrm{m})$ is valid for all the images. 


\subsection{Cell differentiation}

The presence of two hyaline cartilage biomarkers as aggrecan and type II collagen was confirmed. The presence of type II collagen was observed at 21 days (figure 7 ) on the amorphous non-oriented PLLA membrane and the same sample demonstrated to have the higher aggrecan production (figure 8). However, no type II collagen was observed in the oriented PLLA membranes.

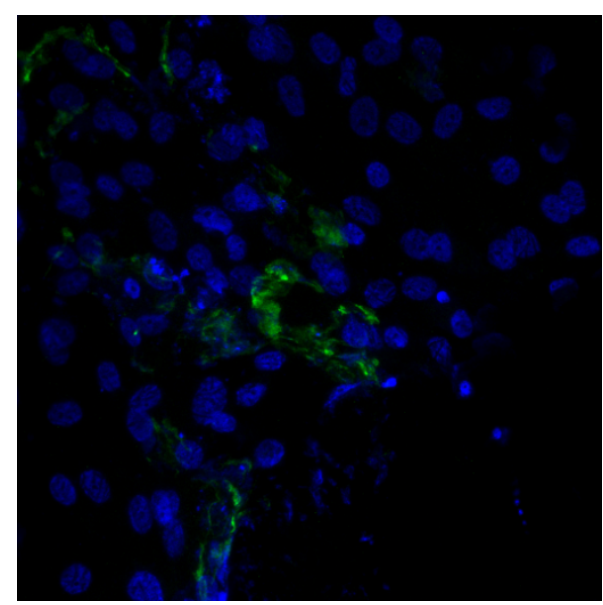

Figure 7 - Immunocytochemical visualization of type II collagen after 21 days of chondrocytes culture in amorphous non-oriented PLLA membrane.

Aggrecan is the most common proteoglycans of the cartilage matrix [24] and its content in supernatant after chondrocyte cells seeded for 7,14 and 28 days is shown in figure 8.

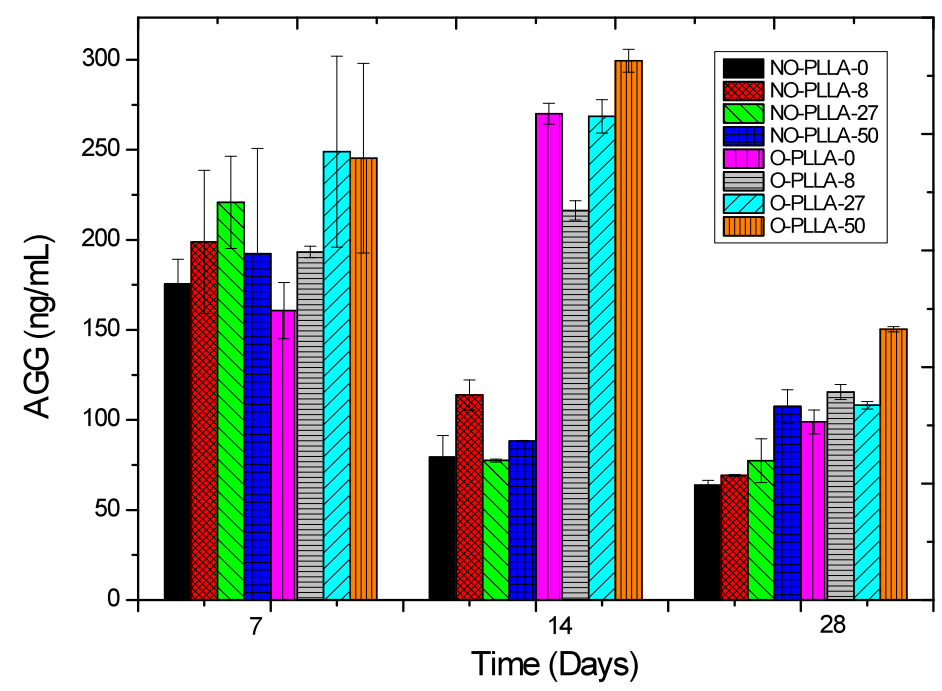

Figure 8 - Human aggrecan production in supernatant after cells seeded for 7, 14 and 28 days on different PLLA membranes. Values are mean \pm SD. No statistically 
significant difference in concentration was found among samples after 7 and 28 days. At 14 days, significant differences between PLLA non-oriented and oriented membranes were found.

At 7 days, no differences are detected in the production of AGG between non-oriented and oriented PLLA membranes. At 14 days it is evident the strong difference between the nonoriented and oriented PLLA membranes. The NO-PLLA-0 shows the lowest AGG production in the supernatant and the O-PLLA-50 the highest. At 28 days, the same behavior was observed with no strong differences between the main groups.

\section{Discussion}

Electrospinning is a powerful technique to produce micro- and nano-fibers and it is know that the different fibrous structures can result in a remarkable increase of surface hydrophobicity [25-27]. The present results show a large increase of water contact angle (WCA) with increasing degree crystallinity in randomly oriented fiber mats and a higher hydrophobicity of the mats with aligned fibers with respect to the former ones.

Highly hydrophobous surfaces can be produced by introducing submicron roughness into an inherently hydrophobous surface [28-30]. Electrospinning is very adequate to generate roughness in the sub-micron range since both fibrils and fiber separation are in this order of magnitude. It should, thus be expected that water contact angle in electrospun mats depends on morphological parameters such as fiber orientation and porosity or fiber diameter [31-33]. The generation of topography in the nanometric scale on the submicron rough surface with an additional decoration of the electrospun fibrils or the space among them produces an increased tendency to water repulsion producing superhydrophobic surfaces. Thus, $\mathrm{Li}$ et al found an important increase of hydrophobicity when combining in a mat polystyrene and nylon6 electrospun fibers with mean diameters quite different from each other [34]. The combination of thin and thick fibers mimics the superhydrophobic surfaces of living organisms such as lotus leaf [25]. Simultaneous contributions of chemical and topographical cues to hydrophobicity can be obtained by coating electrospun fibers with more hydrophobic polymers [35]. Pisuchpen et al obtained mats with WCA above $150^{\circ}$ with poly(vinyl alcohol) fibers coated with or silica or silanol nanoparticles. WAC as high as $178^{\circ}$ were obtained with electrospun polystyrene or poly(vinyl chloride) incorporating nanoparticles that modify 
fibril surface roughness [36], or WAC above $140^{\circ}$ in poly(vinylidene fluoride) containing silica nanoparticles [37]. On the other hand the air retention at the surface also contribute to water repellency, an increase of the stiffness has been shown to favor air retention at the surface, too [38].

The development of crystallinity in PLLA films due to thermal treatments produces a surface roughness that highly depends on the size of the spherulites [39-40]. It is interesting to notice at this point that the variations in the degree of crystallinity in PLLA films does not imply significant variations either in the contact angle $\left(74^{\circ}+/-3\right.$ for $10 \%$ and $67 \%$ crystalline samples) [41] nor in the stiffness (Young Modulus of $3.4+/-0.3$ and $3.5+/-0.2 \mathrm{GPa}$ for $7 \%$ and $40-50 \%$ crystalline samples) [42]. When the amorphous electrospun fibrils crystallize their stiffness and surface energy increase [22, 43], and at the same time surface nano-roughness is expected to be developed.

The combination of all these effects have as a consequence the clear increase of water repellence of the mat surface. The effect is very apparent in randomly oriented fiber mats as the WCA is quite low, much smaller than in the mats with aligned fibers. The results reported in the literature with respect to the effect of fiber alignment are contradictory. Wang et al found higher WCA in randomly oriented electrospun PLLA surfaces than in aligned ones [44], similar result was obtained by Kai et al in electrospun polycaprolactone, although significant increase of WCA with alignment was found when the fibers were modified with gelatin [45-46]. In our case the high hydrophobicity of the mats with aligned fibers (values of WCA of $120^{\circ}$ for the amorphous or $140^{\circ}$ for the most crystalline mats) could be mainly due to the difference in the average pore size (or inter-fiber distance) with respect to randomly oriented mats that can be someway characterized by membrane porosity which is higher in randomly oriented mats [22]. The effect of the roughness introduced by crystallization seems to less important than in non-aligned samples although still significant.

The topography created by the membrane features has a strong influence on the biological response of the cells seeded in its surface trough contact guidance. In spite of the porosity of the electrospun mats, they must be considered as a two dimensional substrates with respect to cell culture since inter-fiber spaces do not allow cell invasion of the three dimensional fiber structure. Cell-substrate interaction mediated by proteins adsorbed from the culture medium is crucial to control chondrocyte adhesion, proliferation and consequently gene expression in monolayer culture [5, 47-48]. It has been shown that nano-fibrilar substrates allow viable and proliferative chondrocyte 
culture $[32,49]$. Mature chondrocytes are non-proliferative cells in hyaline cartilage. The first stage for chondrocytes proliferation when they are seeded on a flat substrate is the recognition by integrins of specific ligands in the proteins adsorbed on the substrate surface. Once adhered to these ligands, integrins cluster and form focal adhesions, which establish links in the cytoplasm with $\alpha$-actin fibers. Development of the actin stress fibers of the cell cytoskeleton not only controls cell morphology but it establishes a cross-talk between the cell nucleus and the extracellular matrix which is an important signaling path regulating cell functions such as proliferation or gene expression. Chondrocytes seeded on a flat PLLA substrate spread and develop well defined focal adhesions and actin cytoskeleton. The peculiar morphology of electrospun mats imposes strong limitations to the formation of focal adhesions. This fact is responsible for the complex morphology of the cells cultured on electrospun mats [50] that can be observed in the SEM pictures of figure 3. Substrate morphology limits cell spreading [39] as can be observed in figure 4. Cells cultured in randomly aligned fiber mats have a non-fully extended morphology although the actin cytoskeleton is well developed (figure 4). Interestingly contact guidance makes that cells seeded in substrates with aligned fibers adopt an elongated morphology that has been observed many times when culturing different cell types on aligned electrospun membranes. Interestingly, the imposed restriction to spreading does not reduce the ability of chondrocytes to proliferate up to 14 days as can be seen by the MTT assays in the amorphous aligned or non-aligned mats. The decrease in viability for longer times is a consequence of the chondrogenic medium, without FBS, employed in cell culture. Nevertheless, cell numbers are still important up to 21 days culture as seen in the live-dead test shown in figure 6 . Interestingly, fiber orientation and the hydrophobicity associated does not seem to affect cell proliferation in the case of amorphous fibers. The values of MTT tests are nearly identical in both supports. Crystallinity seems to decrease proliferation in the nonoriented supports, although dependence on crystallinity is not completely systematic. Proliferation drops sharply with the development of crystallinity in aligned fibers. It seems that proliferation is nearly suppressed when a limit in WCA is attained. BallesterBeltran et al [28] have recently shown that superhydrophobous surfaces can hinder the formation of focal adhesion. Suppression of proliferation in chondrocyte culture seems to be a requirement for the expression of the genes characteristic of the chondrocytes of hyaline cartilage and production of extracellular matrix. Interestingly, supports with crystalline aligned fibers induce active production of aggrecan by cells, collagen type II 
markers are positive only for several cells (figure 7). Lim et al showed good performance of highly hydrophopbous electrospun fiber mats in tissue production of bovine chondrocytes as well $[32,49,51]$.

\section{Conclusions}

PLLA electrospun fibers are nearly amorphous after the electrospinning process. Aligned fiber mats are much more hydrophobous than randomly aligned ones. It is not possible to conclude that the fact that the fibers are aligned parallel to each other is crucial for this repellency to water. The inter-fiber void size can be also important and as detected by the difference in porosity, inter-fiber spacing is smaller in aligned fibers. Crystallization of the fibrils by thermal annealing at temperatures below melting does not affect membrane morphology, but produce an important increase of hydrophobicity what is ascribed to the modification of the fiber roughness at nanometric scale and increase in fiber stiffness. Proliferation of human chondrocytes cultured in monolayer on these substrates is not different in aligned or non-aligned amorphous mats. Crystallization of the aligned mats nearly suppresses proliferation and the cells produce higher amounts of aggrecan, characteristic of the extracellular matrix of hyaline cartilage. The presence of collagen type II is only detected around part of the cultured cells, what is not unexpected in a monolayer culture even if culture medium is chondrogenic.

\section{Acknowledgements}

This work is funded by FEDER funds through the "Programa Operacional Factores de Competitividade - COMPETE" and by national funds by FCT- Fundação para a Ciência e a Tecnologia, project references PTDC/CTM/69316/2006, PTDC/CTMNAN/112574/2009, and NANO/NMed-SD/0156/2007. V.S. thanks the FCT for the SFRH/BPD/63148/2009 grant. C. R. thanks the IINL for a financial support via a PhD grant. NG thanks to Red Temática de Investigación Cooperativa en Envejecimiento y Fragilidad (RETICEF), which is initiative of the ISCIII and the Catalan Government (2009SGR00848). JLGR acknowledge the support of the Spanish Ministry of Education through project No. MAT2010-21611-C03-01 (including the FEDER 
financial support). The authors thank the support from the COST Action MP1003, 2010, 'European Scientific Network for Artificial Muscles’ (ESNAM).

\section{References}

1. Huebsch, N. and D.J. Mooney, Inspiration and application in the evolution of biomaterials. Nature, 2009. 462(7272): p. 426-432.

2. Place, E.S., N.D. Evans, and M.M. Stevens, Complexity in biomaterials for tissue engineering. Nat Mater, 2009. 8(6): p. 457-470.

3. Rotter, N., et al., Cartilage tissue engineering using resorbable scaffolds. Journal of Tissue Engineering and Regenerative Medicine, 2007. 1(6): p. 411416.

4. Vasita, R. and D.S. Katti, Nanofibers and their applications in tissue engineering. International Journal of Nanomedicine, 2006. 1(1): p. 15-30.

5. Kim, D.-H., et al., Biomimetic Nanopatterns as Enabling Tools for Analysis and Control of Live Cells. Advanced Materials, 2010. 22(41): p. 4551-4566.

6. \#246, et al., Proteoglycan Biosynthesis during Chondrogenic Differentiation of Mesenchymal Stem Cells. TheScientificWorldJOURNAL, 2007. 7: p. 12071210.

7. Szivek, J.A., et al., Selective cell proliferation can be controlled with CPC particle coatings. Journal of Biomedical Materials Research Part A, 2007. 81A(4): p. 939-947.

8. Izquierdo, R., et al., Biodegradable PCL scaffolds with an interconnected spherical pore network for tissue engineering. Journal of Biomedical Materials Research Part A, 2008. 85A(1): p. 25-35.

9. Yaszemski, M.J., et al., Evolution of bone transplantation: molecular, cellular and tissue strategies to engineer human bone. Biomaterials, 1996. 17(2): p. 175185.

10. Murugan, R. and S. Ramakrishna, Nano-Featured Scaffolds for Tissue Engineering: A Review of Spinning Methodologies. Tissue Engineering, 2006

12(3): p. 435-447

11. Chen, J.-P. and C.-H. Su, Surface modification of electrospun PLLA nanofibers by plasma treatment and cationized gelatin immobilization for cartilage tissue engineering. Acta Biomaterialia, 2011. 7(1): p. 234-243.

12. Lampin, M., et al., Correlation between substratum roughness and wettability, cell adhesion, and cell migration. Journal of Biomedical Materials Research, 1997. 36(1): p. 99-108.

13. Anselme, K., et al., The interaction of cells and bacteria with surfaces structured at the nanometre scale. Acta Biomaterialia, 2010. 6(10): p. 38243846.

14. Martínez, E., et al., Effects of artificial micro- and nano-structured surfaces on cell behaviour. Annals of Anatomy - Anatomischer Anzeiger, 2009. 191(1): p. 126-135.

15. Yim, E.K.F. and K.W. Leong, Significance of synthetic nanostructures in dictating cellular response. Nanomedicine: Nanotechnology, Biology and Medicine, 2005. 1(1): p. 10-21. 
16. Yang, Y. and K.W. Leong, Nanoscale surfacing for regenerative medicine. Wiley Interdisciplinary Reviews: Nanomedicine and Nanobiotechnology, 2010. 2(5): p. 478-495.

17. Jeon, H., et al., The effect of micronscale anisotropic cross patterns on fibroblast migration. Biomaterials, 2010. 31(15): p. 4286-4295.

18. Yim, E.K.F., S.W. Pang, and K.W. Leong, Synthetic nanostructures inducing differentiation of human mesenchymal stem cells into neuronal lineage. Experimental Cell Research, 2007. 313(9): p. 1820-1829.

19. Gümüşderelioğlu, M., F. Betül Kaya, and I.G. Beşkardeş, Comparison of epithelial and fibroblastic cell behavior on nano/micro-topographic PCL membranes produced by crystallinity control. Journal of Colloid and Interface Science, 2011. 358(2): p. 444-453.

20. Ma, Z., et al., Chondrocyte behaviors on poly-l-lactic acid (PLLA) membranes containing hydroxyl, amide or carboxyl groups. Biomaterials, 2003. 24(21): p. 3725-3730.

21. Cui, Y.L., et al., Biomimetic surface modification of poly (L-lactic acid) with gelatin and its effects on articular chondrocytes in vitro. Journal of Biomedical Materials Research Part A, 2003. 66A(4): p. 770-778.

22. Ribeiro, C., et al., Tailoring the morphology and crystallinity of poly(L-lactide acid) electrospun membranes. Science and Technology of Advanced Materials, 2011. 12(1).

23. Pérez Olmedilla, M., et al., Response of human chondrocytes to a non-uniform distribution of hydrophilic domains on poly (ethyl acrylate-co-hydroxyethyl methacrylate) copolymers. Biomaterials, 2006. 27(7): p. 1003-1012.

24. Rahman, M.S. and T. Tsuchiya, In vitro culture of human chondrocytes (1): A novel enhancement action of ferrous sulfate on the differentiation of human chondrocytes. Cytotechnology, 2001. 37(3): p. 163-169.

25. Wang, X.F., et al., Engineering biomimetic superhydrophobic surfaces of electrospun nanomaterials. Nano Today, 2011. 6(5): p. 510-530.

26. Kong, J., et al., Wettability of Polyethylene Micropatterns with Aligned OneDimensional Nanostructures. Journal of Macromolecular Science Part BPhysics, 2010. 49(4): p. 711-722.

27. Chen, H.C., C.H. Tsai, and M.C. Yang, Mechanical properties and biocompatibility of electrospun polylactide/poly(vinylidene fluoride) mats. Journal of Polymer Research, 2011. 18(3): p. 319-327.

28. Ballester-Beltran, J., et al., Role of superhydrophobicity in the biological activity of fibronectin at the cell-material interface. Soft Matter, 2011. 7(22): p. 1080310811.

29. Erbil, H.Y., et al., Transformation of a simple plastic into a superhydrophobic surface. Science, 2003. 299(5611): p. 1377-1380.

30. Shi, L., et al., Effect of additives on the fabrication of poly (vinylidene fluorideco-hexafluropropylene) (PVDF-HFP) asymmetric microporous hollow fiber membranes. Journal of Membrane Science, 2008. 315(1-2): p. 195-204.

31. Cho, D., et al., Structural properties and superhydrophobicity of electrospun polypropylene fibers from solution and melt. Polymer, 2010. 51(25): p. 60056012.

32. Lim, G.T., et al., Highly Hydrophobic Electrospun Fiber Mats from Polyisobutylene-Based Thermoplastic Elastomers. Biomacromolecules, 2011. 12(5): p. 1795-1799. 
33. Zheng, J., et al., Studies on the controlled morphology and wettability of polystyrene surfaces by electrospinning or electrospraying. Polymer, 2006. 47(20): p. 7095-7102.

34. Li, X.H., et al., Enhanced Mechanical Properties of Superhydrophobic Microfibrous Polystyrene Mats via Polyamide 6 Nanofibers. Journal of Physical Chemistry C, 2009. 113(47): p. 20452-20457.

35. Ma, M.L., et al., Superhydrophobic fabrics produced by electrospinning and chemical vapor deposition. Macromolecules, 2005. 38(23): p. 9742-9748.

36. Asmatulu, R., M. Ceylan, and N. Nuraje, Study of Superhydrophobic Electrospun Nanocomposite Fibers for Energy Systems. Langmuir, 2010. 27(2): p. 504-507.

37. Wang, S., et al., Preparation of a durable superhydrophobic membrane by electrospinning poly (vinylidene fluoride) (PVDF) mixed with epoxy-siloxane modified SiO2 nanoparticles: A possible route to superhydrophobic surfaces with low water sliding angle and high water contact angle. Journal of Colloid and Interface Science, 2011. 359(2): p. 380-388.

38. Pisuchpen, T., et al., Tuning Hydrophobicity and Water Adhesion by Electrospinning and Silanization. Langmuir, 2011. 27(7): p. 3654-3661.

39. Costa Martinez, E., et al., Effect of poly(L-lactide) surface topography on the morphology of in vitro cultured human articular chondrocytes. Journal of Materials Science-Materials in Medicine, 2007. 18(8): p. 1627-1632.

40. Costa Martinez, E., et al., Human Chondrocyte Morphology, Its Dedifferentiation, and Fibronectin Conformation on Different PLLA Microtopographies. Tissue Engineering Part A, 2008. 14(10): p. 1751-1762.

41. Park, A. and L.G. Cima, In vitro cell response to differences in poly-L-lactide crystallinity. Journal of Biomedical Materials Research, 1996. 31(1): p. 117-130.

42. Sarasua, J.R., et al., Crystallinity and mechanical properties of optically pure polylactides and their blends. Polymer Engineering and Science, 2005. 45(5): p. 745-753.

43. Salgado, A.J., et al., Influence of molecular weight and cristallinity of poly(Llactic acid) on the adhesion and proliferation of human osteoblast like cells, in Advanced Materials Forum Iii, Pts 1 and 2, P.M. Vilarinho, Editor. 2006. p. 1020-1024.

44. Wang, B., et al., The effect of poly (L-lactic acid) nanofiber orientation on osteogenic responses of human osteoblast-like MG63 cells. Journal of the Mechanical Behavior of Biomedical Materials, 2011. 4(4): p. 600-609.

45. Kai, D., et al., Guided orientation of cardiomyocytes on electrospun aligned nanofibers for cardiac tissue engineering. Journal of Biomedical Materials Research Part B-Applied Biomaterials, 2011. 98B(2): p. 379-386.

46. Feng, L., et al., Super-hydrophobic surface of aligned polyacrylonitrile nanofibers. Angewandte Chemie-International Edition, 2002. 41(7): p. 1221-+.

47. Torres, A.J., et al., Nanobiotechnology and Cell Biology: Micro- and Nanofabricated Surfaces to Investigate Receptor-Mediated Signaling. Annual Review of Biophysics, 2008. 37(1): p. 265-288.

48. Zhang, X., et al., Nanofiber-modified surface directed cell migration and orientation in microsystem. Vol. 5. 2011: AIP. 032007.

49. Li, W.J., et al., Biological response of chondrocytes cultured in threedimensional nanofibrous poly(epsilon-caprolactone) scaffolds. Journal of Biomedical Materials Research Part A, 2003. 67A(4): p. 1105-1114. 
50. Luong, T.H.N., et al., The role of nanofibrous structure in osteogenic differentiation of human mesenchymal stem cells with serial passage. Nanomedicine, 2011. 6(6): p. 961-974.

51. Subramanian, A., et al., Preparation and evaluation of the electrospun chitosan/PEO fibers for potential applications in cartilage tissue engineering. Journal of Biomaterials Science-Polymer Edition, 2005. 16(7): p. 861-873. 